\title{
Improving the Physical Health Monitoring of City \& Hackney Assertive Outreach Service Patients
}

\author{
Elvan Akyuz, amit jain, declan phelan, Susham Gupta
}

East London NHS Foundation Trust

\begin{abstract}
Improving physical healthcare to reduce premature mortality in people with SMI (Serious Mental Illness) is a priority for ELFT (East London NHS Foundation Trust) and NHS England. It is well know that people with schizophrenia have a life expectancy which is approximately $20 \%$ shorter than that of the general population and a substantial mortality difference exists between people with schizophrenia and the general community.[1-2] Among other risk factors, such as poor diet, physical inactivity, and high rates of smoking, the iatrogenic effects of antipsychotic medications have been found to increase the risk of metabolic syndrome. This can easily be detected through regular monitoring.

Through this project, it was our aim to improve the physical health monitoring of City \& Hackney Assertive Outreach Service (AOS) patients with a view to decrease mortality rate, increase life expectancy, increase the quality of life, and reduce harm from medication. This was done using quality improvement methods, including several change ideas, each of which started sequentially over the course of a nine month period from November 2014. Following QI methodology, this utilised cycles of iterative learning using PDSA methods and was supported by the Trust's extensive programme of quality improvement, including training provided by the Institute for Healthcare Improvement.
\end{abstract}

The project involved setting a specific aim which was improving the physical health monitoring of AOS patients to $80 \%$ by July 2015 and for our patients to have physical health checks (blood tests, weight, ECG, BP) as a minimum annually. From baseline measurements of between $50-75 \%$, we reached our target of $80 \%$ for weight, BP and blood tests monitoring, with $89 \%, 91 \%$, and $84 \%$ achieved respectively by July 2015. Further progress still needs to be made on ECGs, with $77 \%$ achieved by July 2015 , although the monitoring of ECG nearly doubled from $39 \%$ in November 2014 to $77 \%$ in July 2015.

This project demonstrated that effective leadership, collaborative team work, embedding the project in our daily practice, generating local solutions, and improved communication between primary and secondary care can increase the physical health monitoring of this complex and challenging patient group. The team continues to identify other areas for change that may lead to further improvement in the physical health monitoring of AOS patients while making sure that the improvement is sustained.

\section{Problem}

Schizophrenia is a disabling and distressing mental health disorder that is also associated with decreased life expectancy due to cardiovascular risk factors such as obesity, diabetes, dyslipidaemia, and hypertension, resulting in a two to threefold increased risk of death and a reduced life span of 12 to 15 years compared with the general population.[3-7]

Second generation antipsychotic agents used in the treatment of schizophrenia may have a negative impact on components of metabolic syndrome such as weight, blood pressure, glucose level, and lipid metabolism, $[8,9]$ thereby having the potential to exacerbate patients' already high risk of cardiovascular disease.

Improving physical healthcare to reduce premature mortality in people with SMI is a priority for ELFT and it is a strategic aim of our Trust. The Trust has already agreed to carry out regular audits supported by the Royal College of Psychiatrists to demonstrate its commitment to improved physical health. One of the notable findings regarding ELFT in the Report of the Second Round of the National Audit of Schizophrenia (NAS2) 2014 was "although monitoring of physical health risk factors were about average in ELFT, it was still below what should be provided and was particularly poor for monitoring of glucose control and lipids".[10]

The inadequate physical health monitoring of SMI patients is a not a problem that is unique to ELFT. In a study in Southampton and Winchester, looking into screening and prevalence of metabolic syndrome in both inpatients and outpatients, found that a considerably low proportion of the patients were screened for metabolic factors; blood pressure (32\%), glucose (16\%), lipids $(9 \%)$, and weight $(2 \%)$ with more than $50 \%$ of the patients fulfilling the criterion for metabolic syndrome who were not screened before.[11] In another study of an assertive outreach teams with patients receiving more intensive input than generic team results were not encouraging with less than $30 \%$ of the patients screened for various metabolic factors.[12]

The project was based in an assertive outreach team which is multidisciplinary, in Hackney, East London. According to the Index of Multiple Deprivation 2010, at local authority district level, Hackney is the most deprived borough in England among 326 local authorities. The team's caseload was around 100, and was made up of patients 
BMJ Quality Improvement Reports

who are challenging to engage, with complex health and social needs. Each patient is care coordinated and receives more intense input compared with generic teams.

\section{Background}

It has been extensively researched that people with SMI are at higher risk of premature death from both natural and unnatural (suicide) causes.[13] People with schizophrenia have a mortality risk that is two to three times that of the general population. Schizophrenia is associated with increased death rates from cardiovascular disease (two-fold), respiratory disease (three-fold), and infectious disease (four-fold), amongst others. Among all other cardiovascular risk factors of poor diet, physical inactivity, and high rates of smoking, anti-psychotic medications have been found to significantly increase the risk of metabolic syndrome.

However, despite the above evidence, physical health needs and monitoring of the people on antipsychotic medication has been lacking and often not adequately addressed both in primary or secondary care settings. $[14,15]$

In the light of the above evidence over the last 15 years, the need for screening, monitoring, and prevention of diabetes and other cardiovascular disease risk factors has been acknowledged and multiple guidelines on physical health monitoring for people with SMI have been recommended. Evaluation of 18 guidelines published between 2004 and 2010 from different countries was similar in recommending variables to be monitored. However, they differ in timing and interval between the assessments.[16] In the UK, three different guidelines are in place, the three key guidelines are: the revised National Institute for Health and Clinical Excellence (NICE) schizophrenia guidelines (2014),[17] Updated Lester Positive Cardio Metabolic Health Resource (2014),[18] and UK Quality and Outcomes Framework (QOF) for Primary Care.[19]

Reductions in mortality and improvements in physical health monitoring in patients with mental health problems are required by the NHS Outcomes Framework and a national commissioning for quality and innovation scheme. $(20,21)$

Factors responsible for shortcomings in physical health monitoring are multi-faceted. These can broadly be understood as patient, professionals and also service related. Guidelines have the potential to "improve the care received by patients by promoting interventions of proven benefit" and to "inform clinical practice, promoting effective and cost effective health care" yet current guidelines for metabolic screening in this patient population seem to have had little impact on clinical practice or patient outcomes (22). A one size fits all approach might not work for all the services and regions, considering their respective service provisos and resources and population demographics.

There is a need to think of small changes or developments to the existing services which are easy and cost effective to trial and, if found effective, implement in order to improve the physical health monitoring of psychiatric patients. The goal of the current quality improvement project was to develop such an approach in assertive outreach patients in East London NHS Foundation Trust.

\section{Baseline measurement}

The overall aim of the project was to improve the physical health monitoring of AOS patients in the community and ensure that every patient was offered psycho-education and encouraged and supported to have physical health checks/monitoring (blood tests including lipid, glucose, ECG, BP, weight with BMI) as a minimum annually and to record it in a structured way using CPA (Care Programme Approach) forms and on the Trust's electronic patient record system RIO, including the reason if it is not undertaken. If not undertaken, the reasons and the steps taken to tackle the reason(s) need to be recorded.

To establish the baseline data we initially opted to ask care coordinators to fill in the physical health form based on what was available on RIO electronic patient notes, after discussing the project at the team meeting several times. However, the initial response rate varied significantly between care coordinators from no response to full response, which highlighted that the care coordinators still need education and active encouragement about the project. From this we learnt that we needed to include the project as part of the weekly team meeting, which led to a $100 \%$ response rate prior to the start of the project.

We were also informed by care-coordinators that there was no straightforward way to find information about physical health monitoring on RIO, as at that time there was no systematic means of recording this information (the Trust has subsequently developed a process and system for this, which is currently being implemented). To enable our understanding of baseline for this project, we therefore decided to request patients' physical health summaries from their GPs. We also felt this would have the added benefit of developing closer working relationships with GPs.

We prepared a template for the summary of the physical health request form for our admin to fax to GP's receptionists. The information we received was recorded on an Excel spreadsheet in alphabetical order. The response rate from GPs was $71 \%$. Weight and BP was similar at $73 \%$ and $72 \%$, respectively, however only $54 \%$ had blood tests. In terms of blood tests, some of them had fasting lipids, some had fasting glucose $\mathrm{HbA} 1 \mathrm{c}$ and some had both, which showed some inconsistencies between practices. Only ten patients had ECG recorded, which we interpreted as an indication of ECGs not being requested by GPs as a part of psychotropic protocol.

To establish our baseline, we combined the data from all of the above sources, which resulted in baselines values of $51 \%$ for weight, $48 \%$ for BP, $40 \%$ for blood test, and $39 \%$ for ECG.

\section{Design}

A local team was established, including medical and management of frontline staff, with the support of the Trust's Quality Improvement team. The design of the project used the model for improvement, 
developed by Associates in Process Improvement and the adopted method of the Institute for Healthcare Improvement. Project leads attended Improvement Science in Action training to gain skills in the model. Having established the multi-disciplinary teams,

opportunities were created to involve GPs and carers in our plans, and a regular meeting time and date was agreed. We put together a driver diagram to reveal the relevant factors we needed to address to meet our aims.

We used baseline data to set a target for improvement, which was improving the physical health monitoring of AOS patients to $80 \%$ by May 2015 and offering/supporting patients to have these measures to $100 \%$. We generated change ideas which included use of CPA forms and RIO codes to record and share the monitoring in a structured way, reminding patients to attend tests and booking joint GP reviews.

It was felt that a vital component of the project would be to raise awareness of the importance of physical health monitoring. Therefore, we established a regular time and regular place to update the team. Each team member was encouraged to identify factors that might be hindering the process of their patient's engagement with physical health monitoring. However, soon after starting, we decided to ring-fence time for a fortnightly 30 minute meeting with the local team to a weekly team meeting, with the aim of keeping the momentum going and recording any action/result at the team meeting minutes.

The data is attributes data, originally collected as a whole number and classified as a percentage. Due to low number of data points, this data is presented as run charts, showing how the percentage of physical health monitoring progressed from November 2014 to July 2015 , by month. This meant that the intervention's effect was tracked over time. In the course of the project this data was provided to the project sponsor and presented at the weekly team meeting every four weeks.

\section{Strategy}

The strategy for implementation of change ideas was based on the PDSA cycle.

Change 1: The name of patients who had any Bloods + ECG since 29th October 2014 to be discussed and recorded at the weekly team meetings to embed it in our daily practice culture. There was an immediate increase in the percentage of patients who had these measures. It was also found to be effective in reducing the missed data and so it was continued.

Change 2: The data including the Excel spreadsheet and the $\mathrm{P}$ chart of the percentage of physical health monitoring was presented at the weekly team meeting every four weeks from the beginning of December 2014 with a view to review the progress and to communicate challenges and to standardize processes in order to ensure understanding of the project. In addition to this, the visual display of the data and progress was been found to be very effective at bringing about improvement.
Change 3: Systematically started allocating patients for support workers and care coordinators to be taken to Homerton University Hospital for ECG and Blood tests from December 2014. It was particularly effective for the patient group, which suffered from the negative symptoms of schizophrenia.

Change 4: Booking joint GP review for patients that have complex physical health needs from December 2014. This practice was a starting point for improved communication and collaborative team work between primary and secondary care, which can increase the physical health monitoring of this complex and challenging patient group.

Change 5: Recording and sharing the monitoring in a structured way using CPA forms and RIO codes (RCODE PHYSH01 is for recording progress notes and for uploading the code is MREP). In mid-January it was decided by the team that ahead of the CPA review, the care coordinator should collate all relevant physical health information, including notes from previous CPA reviews and annual health check results from GP. If an annual health check has not been carried out, care coordinators should organise baseline checks in the appropriate service and make sure that CPA outcome letter and care plan is shared with GP.

Change 6: Test using physical health monitoring pod machines (which enable patients to easily check and print their own height, weight, and blood pressure) at the end of January 2015 as a part of the pilot study. The percentage of patients that used the pod was $86 \%$ which was quite impressive. However, it was noted that almost all the patients who used the pod were encouraged personally by the staff members to use the pod, despite the clear instructions posted on the wall requesting them to use it, as it was reported by them that they did not feel confident to use it first time but most of the patients agreed to use it next time by themselves. Feedback from majority of the patients on experience of using the pod was positive and they also found it very helpful to discuss their physical health. A few female patients were worried about privacy, and stated that they would use if there was a screen or if pod was in a more private location.

Change 7: Group spreadsheet by Allotted Care Coordinator to make it easier for care coordinators to have control over their cases and to indicate how they were performing and also to create healthy competition to help drive improvement.

\section{Results}

Overall, there were significant improvements in the first three months of the project, from November 2014 to the end of January 2015 (resulting from change Ideas 1-4), with an improvement of approximately $50 \%$ in all categories. By June 2015 , the project achieved its aim of physical health monitoring in $80 \%$ of patients in terms of weight, blood pressure, and blood tests. Further progress still needs to be made on ECGs, with $77 \%$ achieved by July 2015 , although the monitoring of ECG nearly doubled from $39 \%$ in November 2014 to $77 \%$ July 2015. By July 2015, each patient was offered physical health monitoring and this was recorded in their RIO notes but there were still difficulties with using RIO Codes and 
staff needed to be reminding via email and/or at the team meeting.

Although it was not within our specific aim for this project, alongside this work we also gathered additional data about physical health status and risk factors in our AOS and also our forensic AOS (the Forensic AOS is not included in the physical health monitoring project and the monitoring data discussed above). Looking at the period November 2014 to May 2015, we established the percentage of people who were overweight or obese (74\%), level of hypertension (20\%), diabetes (24\%), hyperlipidaemia (28\%), abnormal ECG (15\%), and the percentage of people who smoked (71\%) in our patient group. With this enhanced understanding, we will now be looking to focus on addressing these risk factors and improving our service users' physical health, through interventions, including health promotion activity.

See supplementary file: ds6452.pptx - "Run Charts_AOS July \& Abnormal Results"

\section{Lessons and limitations}

Even though this project made us realise that the complexities of physical health monitoring of SMI patients are more challenging than at first thought; it demonstrated that effective leadership, collaborative team work, routine recording of the results, and improved communication between primary and secondary care can increase the physical health monitoring of this complex and challenging patient group.

Although the creation of a local database, which is updated at weekly clinical team meetings, and updating the team monthly on the process helped embed this project into daily clinical practice, we learned from February 2015 data that when the project lead was away the rate of improvement relative to previous and subsequent months had declined, therefore, we realised that we need to continue to ensure that this project is owned by the whole team, and all staff are closely kept up-to-date with the process and assisted to continue to deliver improvement, as well as assigning a temporary project lead whilst the project lead is away.

We also learned that Pod machines can be used as a helpful screening tool in the clinical settings. Patient feedback about the use of Pod has been quite positive. Most of the patients would use the Pod but may need initial encouragement from the clinical staff. Although it runs a small risk of false positives, it was argued as one of the cost effective ways to measure physical health parameters and is now a longer term fixture in the services with pod machines in three different sites within the Trust.

We addressed a problem that was important to our Trust, team, staff, carers and patients, and the team was enthusiastic about the project, testing ideas and including everyone actively to bring a change with a view to it leading to improvement. We had strategic support for the project from the directorate, and the technical and methodological support of the quality improvement team. Our QI project lead/manager took the approach of acknowledging the hard work of the team/team members via emails or in the weekly team meetings, in keeping with principles of Ql; believing that you will be more successful if you encourage and reward good practice than to punish bad.

The intervention was cost-effective, as it involved very little financial outlay. The biggest potential source of cost would be the time taken for doctors, care coordinators, and support workers to engage patients in physical health monitoring and take them to either GP surgeries or general hospitals. It was beyond the scope of this project to do further analysis on cost-benefit, but this would be a useful area of study for future work in this area.

Following this project, although the team achieved its aim, the team feels that increasing physical health monitoring of its patients has implications on City \& Hackney AOS's operational functioning, training, and supporting infrastructure, e.g., establishing part-time physical wellness clinic attached to the community teams and/or extending clozapine, depot clinic, and a clear understanding between the Trust and CCGs around shared care protocols such as a shared IT platform for primary care and mental health services.

A limitation of the current project was that it was only implemented in this structure on the AOS team at ELFT. Therefore, at the present time, it is not clear whether the findings generated would be replicated if the change ideas were tested on other mental health community teams with a similar to the structure, where each patient is care coordinated and provided intense support in the community, e.g., Early Intervention in Psychosis, Rehabilitation Psychiatry. There are currently movements to test the intervention more widely within East London Foundation Trust.

\section{Conclusion}

The premature mortality in people with SMI is well known worldwide and improving physical healthcare monitoring to reduce mortality in these group is a priority for ELFT and NHS England. Through this project, we aimed to improve the physical health monitoring of City \& Hackney AOS -Assertive Outreach Service patients. The project benefited from an enthusiastic, motivated, and creative team, which was supported hugely by East London NHS Foundation Trusts' quality improvement team and directorate.

Following a series of interventions aimed at supporting patients having these measures and increasing effective leadership, collaborative team work, and routine recording of the results, as well as improving communication between primary and secondary care and embedding this project into daily clinical practice, overall there has been gradual increase in the physical health monitoring of AOS patients and we have reached our target of $80 \%$ for weight, BP and blood tests monitoring, with $89 \%, 91 \%$, and $84 \%$ achieved respectively by July 2015 . We just fell short of reaching our target for the monitoring of ECGs, which reached $77 \%$ by July 2015 , however major progress was also made in this area, nearly doubling from November 2014 to July 2015.

We believe that the success of this project lay in the enthusiasm of the team, involvement of staff in every step of the project and generating local solutions. The team continues to identify other areas for change that may lead to further improvement in the 
physical health monitoring of AOS patients while making sure that the improvement is sustained. We are embedding many of the interventions we tested into daily practice and continuing to generate new ideas for testing.

\section{References}

1. Newman S.C., Bland R.C. Mortality in a cohort of patients with schizophrenia: a record linage study. Canadian Journal of Psychiatry. 1991;36 (4): 239-245.

2. Brown S. Excess mortality of schizophrenia: a metaanalysis. Br J Psychiatry. 1997; 171:502-508.

3. NICE. Psychosis and schizophrenia in adults: treatment and management. Clinical Guideline 178. NICE, 2014.

4. Saha S, Chant D, McGrath J. A Systematic Review of Mortality in Schizophrenia: Is the Differential Mortality Gap Worsening Over Time? Arch Gen Psychiatry. 2007;64(10):1123-1131.

5. McGrath J, Saha S, Chant D et al. Schizophrenia: a concise overview of incidence, prevalence, and mortality. Epidemiol Rev. 2008; 30:67-76.

6. Chwastiak L. A., Tek C. The unchanging mortality gap for people with schizophrenia. The Lancet. 2009; 374, 9690: 590-592.

7. Van Os J, Kapur S. Schizophrenia. The Lancet. 2009; 374, 9690: 635-645.

8. De Hert M, Schreurs V, Vancampfort D et al. Metabolic syndrome in people with schizophrenia: a review. World Psychiatry. 2009; 8 (1):15-22.

9. Rummel-Kluge C, Komossa K, Schwarz S et al. Head-tohead comparisons of metabolic side effects of second generation antipsychotics in the treatment of schizophrenia: a systematic review and meta-analysis. Schizophr Res. 2010; 123,225-233.

10. Report of the second round of the National Audit of Schizophrenia (NAS2) 2014. Royal College of Psychiatrists. College centre for quality improvement.

11. Holt RI, Abdelrahman T, Hirsch M et al. The prevalence of undiagnosed metabolic abnormalities in people with serious mental illness. J Psychopharmacol 2010; 24:867-73.

12. Barnes TR, Paton C, Cavanagh MR, Hancock E, Taylor DM. A UK audit of screening for the metabolic side effects of antipsychotics in community patients. Schizophr Bull. 2007; 33: $1397-403$.

13. Harris EC, Barraclough B. Excess mortality of mental disorder. Br J Psychiatry. 1998; 173: 11-53.

14. Marder SR, Essock SM, Miller AL, Buchanan RW, Casey DE, Davis JM,et al. Physical health monitoring of people with schizophrenia. Am J Psychiatry 2004; 161: 1334-49.

15. Paton $\mathrm{C}$ et al. Obesity, dyslipidaemias and smoking in an in patient population treated with antipsychotic drugs. Acta Psychiatrica Scandinavica.2004; 110:299-305.

16. De Hert et al. Guidelines for screening and monitoring of cardio metabolic risk in schizophrenia: systematic evaluation. Br J Psychiatry.2011; 199:99-105.

17. NICE clinical guideline 178-Psychosis and schizophrenia in adults: treatment and management. guidance.nice.org.uk/cg178 Issued: February 2014 last modified: March 2014.

18. Lester UK Adaptation 2014 update-Positive Cardiometabolic Health Resource-

www.rcpsych.ac.uk/quality/NAS/resources

19. NHS England. Quality and Outcomes Framework guidance for General Medical Services (GMS) contract 2013/14.

20. Department of Health. NHS outcomes framework 2014/15. London: DH, 2013.

21. Department of Health. Commissioning for quality and innovation (CQUIN) 2014/15 guidance. London: DH, 2013.

22. Grimshaw JM, Thomas RE, MacLennan G, et al. Effectiveness and efficiency of guideline dissemination and implementation strategies. Health Technol Assess. 2004; 8(6): iii-iv

\section{Declaration of interests}

Nothing to declare.

\section{Acknowledgements}

We would like to thank the staff at City \&Hackney AOS Team, Quality Improvement team, General Adult Mental Health Care Directorate of East London NHS Foundation Trust for their contribution to this work, including Jen Taylor-Watt, Tsana Rawson, James Innes \& Amar Shah (QI team), and Toby Baldwin (IT advice).

\section{Ethical approval}

This work was considered exempt from ethical approval as it was deemed an improvement study and not a study on human subjects. 